\title{
Evaluation and Training of Human Finger Tapping Movements
}

\author{
Keisuke Shima ${ }^{1}$, Toshio Tsuji ${ }^{1}$, Akihiko Kandori², \\ Masaru Yokoe ${ }^{3}$ and Saburo Sakoda ${ }^{3}$ \\ ${ }^{1}$ Graduate School of Engineering, Hiroshima University, \\ ${ }^{2}$ Advanced Research Laboratory, Hitachi Ltd, \\ ${ }^{3}$ Graduate School of Medicine, Osaka University \\ Japan
}

\section{Introduction}

The number of patients suffering from motor dysfunction due to neurological disorders or cerebral infarction has been increasing in an aging society. A survey by the Ministry of Health, Labor and Welfare in Japan revealed that the total number of patients with cerebrovascular disease is as high as approximately 137 million people [1]. In particular, Parkinson's disease (PD) is a progressive, incurable disease that affects approximately one in five hundred people (around 120,000 individuals) in the UK [2]. Assessment of its symptoms through blood tests or clinical imaging procedures such as computed tomography (CT) scanning and magnetic resonance imaging (MRI) cannot fully determine the severity of the disease. Evidence obtained from clinical semiology and the assessment of drug therapy efficacy therefore depend on the doctor's inquiries into the patient's status, or on complaints from patients themselves. For patients with such motor function impairment, it is necessary to detect the disease in its early stages by evaluation of motor function and retard its progression through movement rehabilitation training.

For assessment of neurological disorders such as PD or spinocerebellar degeneration, various assessment methods have been used including hand open-close movement, pronosupination and finger tapping movement [3]. In particular, finger tapping movements have been widely applied in clinical environments for evaluation of motor function since Holmes [4] proved that the rhythm of the movements acts as an efficient index for cerebellar function testing. The Unified Parkinson's Disease Rating Scale [3] part III (Motor) finger tapping score (UPDRS-FT) is generally used to assess the severity of PD in patients. However, this method is semiquantitative, and has drawbacks including the vagueness of the basis of evaluation for determining the course of the disease [5]. It would therefore be more practical if clinical semiology and the efficacy of drug therapy could be evaluated easily and quantitatively from finger tapping movements.

The quantification of finger tapping movements has already been extensively investigated through techniques such as evaluating tapping rhythms using electrocardiographic apparatus [6] and examining the velocity and amplitude of movements based on images 
measured by infrared camera [7], [8]. However, Shimoyama et al. [6] discussed the finger tapping rhythms only. These camera systems can capture the 3D motion of fingers, but require large and expensive equipments. Further, a compact, lightweight acceleration sensor [9], [10] and magnetic sensor [11], [12] have been utilized for movement analysis in recent years. As for the evaluation of finger tapping movements, however, only the basic analyses have been performed such as verification of the feature quantities of PD patients, which have never been used for the routine assessment of PD in clinical environments.

Motor function training has also been widely applied in clinical environments, and several efficient training methods have been reported [13]-[15]. As an example, Thaut et al. and Enzensberger et al. conducted walking training along with indicated rhythm or melody for patients recovering from strokes or those with PD. They confirmed that freezing of gait was decreased, and walking velocity and length of stride were increased. Furthermore, Olmo et al. discussed the effectiveness of training for PD patients using finger tapping movements in recent years [15]. Unfortunately, however, the psychological burden on the subjects was a concern due to the one-sided nature of the training, as the trainees must remain under the constant direction of the therapist and the training system. It is therefore necessary to develop a method that can lower the psychological burden and allow the trainee to enjoy the training process to enable training to be continued in daily life.

In this Chapter, we explain a novel evaluation and training method of finger tapping movements to realize a system to support diagnosis and enjoyable motor function training for use in daily life. This system measures finger movements with high accuracy using magnetic sensors [11] developed by Kandori et al. Ten evaluation indices consisting of feature quantities extracted on the basis of medical knowledge (such as the maximum amplitude of the measured finger taps and variations in the tapping rhythm) are computed, and radar charts of the evaluation results are then displayed in real time on a monitor. At the same time, the extracted features are discriminated using a probabilistic neural network (PNN) and allocated as operation commands for machines such as domestic appliances and a game console. The system not only allows users to train finger movements through operation of these machines, but also enables quantitative evaluation of motor functions. The user can therefore intuitively understand the features of finger tapping movements and training results.

In this Chapter, the structure and algorithm of the evaluation and training method for finger tapping movements are explained in Section 2. Sections 3 and 4 describe the experiments conducted to identify the effectiveness of the method. Finally, Section 5 concludes the Chapter and discusses the research work in further detail. 


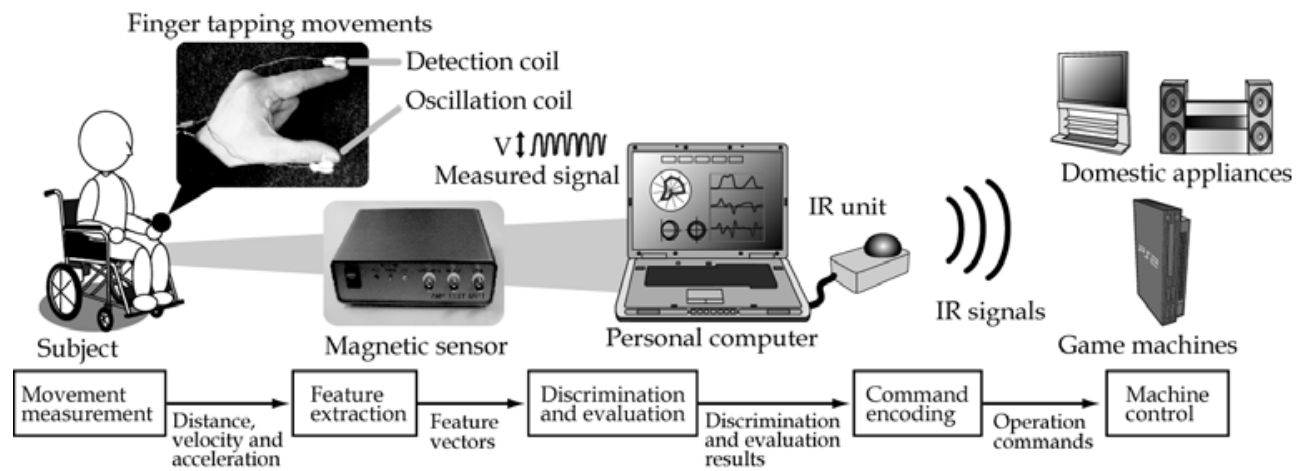

Fig. 1. Overview of the evaluation and training system for finger tapping movements

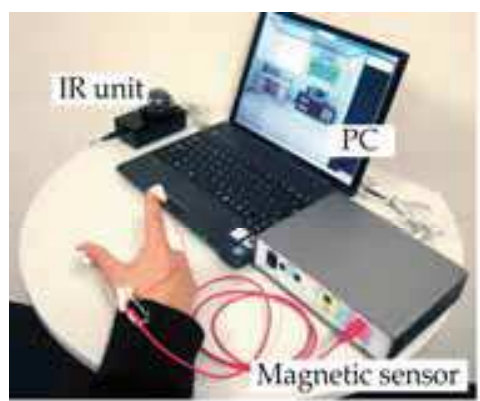

(a) Prototype system developed

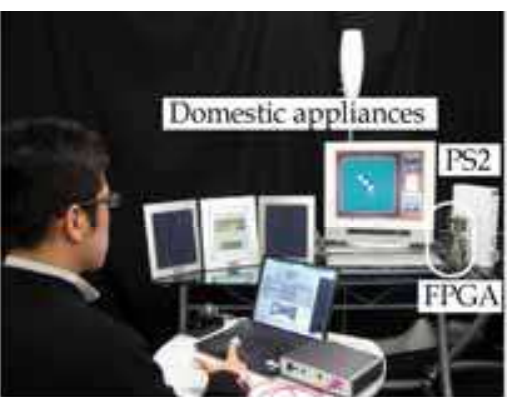

(b) An operation scene using the prototype

Fig. 2. Photographs of the prototype system developed and an operation scene

\section{Evaluation and training system for finger tapping movements}

The measurement and evaluation system of finger tapping movements is shown in Fig. 1. It consists of a magnetic sensor for measuring finger taps and a personal computer (PC). The user conducts finger tapping movements with two magnetic sensor coils attached to the distal parts of the thumb and index finger, and the magnetic sensor then outputs voltages according to the distance between the two coils. The voltages measured are converted into values representing the distance between the two fingertips (the fingertip distance) based on a nonlinear calibration model in the PC. Further, the features of the movements measured are computed from the fingertip distance, velocity and acceleration for evaluation of the finger taps. The details of each process are explained in the following subsections. Figure 2 shows (a) the prototype developed and (b) the operation scene of Othello using the prototype.

\subsection{Magnetic measurement of finger tapping movements [23]}

In this system, the magnetic sensor developed by Kandori et al. [11] is utilized to measure finger tapping movements. The sensor can output a voltage corresponding to changes in distance between the detection coil and the oscillation coil by means of electromagnetic induction. First, the two coils are attached to the distal parts of the user's fingers, and finger 


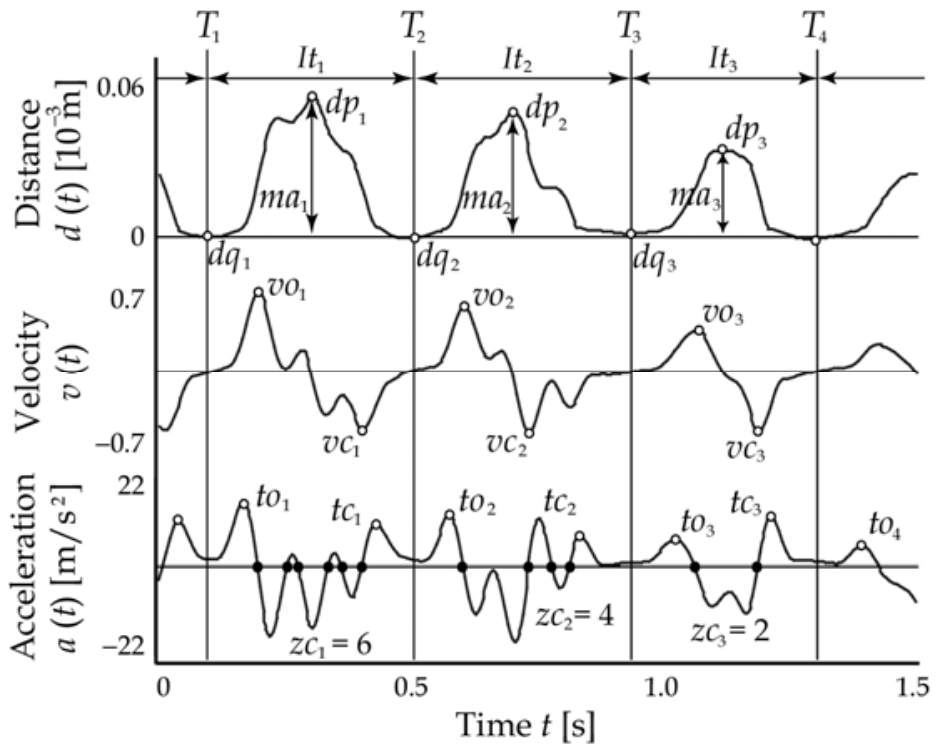

Fig. 3. Examples of the signals measured

tapping movements are measured. The fingertip distances are then obtained from the output voltage by a calibration model expressed as

$$
\begin{aligned}
& d(t)=\alpha^{\prime} \widetilde{V}(t)-\varepsilon, \\
& \widetilde{V}(t)=V^{-\frac{1}{3}}(t),
\end{aligned}
$$

where $d(t)$ denotes the fingertip distance, $V(t)$ is the measured voltage of the sensors at a given time $t$, and $\alpha$ and $\varepsilon$ are constants computed from the calibration [12]. In the calibration process, $\alpha$ and $\varepsilon$ are estimated using the linear least-square method for $n$ values measured output voltages and the fingertip distances of each subject. The calibration process can reduce the influence of the slope of the coils and modeling errors. Further, the velocity $v(t)$ and acceleration $a(t)$ can be calculated from the fingertip distance $d(t)$ using differentiation filters [21].

\subsection{Feature extraction [23]}

The evaluation indices of finger tapping movements are calculated for quantitative evaluation at the feature extraction stage. This Chapter defines ten indices based on previous observations [9], [10] as follows:

(1) Total tapping distance

(2) Average maximum amplitude of finger taps

(3) Coefficient of variation (CV) of maximum amplitude

(4) Average finger tapping interval

(5) CV of finger tapping interval 
(6) Average maximum opening velocity

(7) CV of maximum opening velocity

(8) Average maximum closing velocity

(9) CV of maximum closing velocity

(10) Average zero-crossing number of acceleration

To calculate the above indices, the contact point between the fingers is determined from $d(t)$, $v(t)$ and $a(t)$. First, the threshold $M^{\text {th }}$ is calculated as

$$
\begin{gathered}
M^{\text {th }}=\left\{\begin{array}{cc}
\tilde{M}^{\text {th }} & \left(\tilde{M}^{\text {th }} \geq \varsigma\right), \\
\varsigma & \left(\tilde{M}^{t h}<\varsigma\right)
\end{array}\right. \\
\tilde{M}^{\text {th }}=\eta\left(\frac{1}{K} \sum_{k=1}^{K} d_{k}^{\max }-\frac{1}{K^{\prime}} \sum_{k^{\prime}=1}^{K^{\prime}} d_{k^{\prime}}^{\min }\right),
\end{gathered}
$$

where $\zeta$ and $\eta$ are constants determined by the minimum and maximum values of all subjects' fingertip distances; $d_{k}^{\max }$ denotes the distance between fingertips at the $k$ th time when $v(t)=0$ and $a(t)<0$ in the measurement time window, and $d_{k^{\prime}}^{\min }$ denotes the same at the $k^{\prime}$ th time when $v(t)=0$ and $a(t)>0$; and $K$ and $K^{\prime}$ are the number of $d_{k}^{\max }$ and $d_{k^{\prime}}^{\min }$, respectively. Then, the $i$ th time at which the distance $d_{k^{\prime}}^{\min }$ falls below the threshold $M^{\text {th }}$ is defined as the contact point $T_{i}(i=1,2, \ldots, I$, where $I$ is the number of contacts between fingertips).

First, the integration of the absolute value of velocity $v(t)$ through the measurement time is signified as the total tapping distance (Index 1). As feature quantities of the $i$ th tapping, the maximum and minimum amplitude points $\left(d p_{i}, d q_{i}\right)$ between the interval $\left[T_{i}, T_{i+1}\right]$ are calculated from the measured fingertip distance $d(t)$, and the average (Index 2) and CV (Index 3 ) of maximum amplitudes $m a_{i}=d p_{i},-d q_{i}$ are computed. Further, the finger tapping interval $I t_{i}$, which is the time interval between two consecutive contacts, is applied as $I t_{i}=$ $T_{i+1}-T_{i}$, and the positive and negative maximum velocity points are defined as the maximum opening velocity $v o_{i}$ and the maximum closing velocity $v c_{i}$ respectively. The averages and $\mathrm{CVs}$ of the finger tapping interval, maximum opening velocity and maximum closing velocity are then computed from all the values of $I t_{i}, v o_{i}$, and $v c_{i}$ (Indices 4-9) respectively.

In addition, $z c_{i}$, which denotes the number of zero crossings of the acceleration waveform $a(t)$, is calculated from each interval between $T_{i}$ and $T_{i+1}$, and the numbers of zero-crossing points of acceleration $z c_{i}$ are defined as the evaluation value of multimodal movements (Index 10). Here, the number of zero crossings $z c_{i}$ increases in accordance with the number of extrema of $v(t)$ in a tap movement. As examples, $z c_{3}=2$ implies a smooth tap, while $z c_{1}=$ 6 or $z c_{2}=4$ would represent a jerky tap (see Fig. 3). Multimodal movements that have several peaks of distance in a single finger tap may be observed in PD patients due to bradykinesia and disturbances in rhythm formation. It is therefore possible to evaluate the smoothness of motion based on the number of zero crossings.

Additionally, the $i$ th input vector $\mathbf{x}(i)=\left[x_{1}(i), \ldots, x_{5}(i)\right]^{\mathrm{T}}$ is defined as $x_{1}(i)=m a_{i}, x_{2}(i)=I t_{i}$, $x_{3}(i)=v o_{i}, x_{4}(i)=v c_{i}$, and $x_{5}(i)=z c_{i}$ for discrimination of finger tapping movements using PNN. 


\subsection{Discrimination and evaluation [23][24]}

The calculated evaluation indices of the subject are normalized based on the indices of normal subjects to enable comparison of the difference in movements. Here, it was observed from the preliminary experimental results that three evaluation indices of PD patients (i.e. average maximum amplitude, maximum opening velocity and maximum closing velocity) were smaller than those of normal elderly subjects. These indices were used to calculate the inverse number for every single tap, and the total tapping distance was converted to its inverse number. Hence, all the indices of PD patients are greater than those of normal elderly subjects.

In this system, the standard normally distributed variables $x_{p}$ are converted to the mean and standard deviations of the tapping data from those of the normal subjects using Eq. 3.

$$
x_{p}=\left(z_{p}-\mu_{p}\right) / \sigma_{p},
$$

Here, $p$ corresponds to the index number, $z_{p}$ is the computed value in each index, and $\mu_{p}$ and $\sigma_{p}$ describe the average and standard deviation of each index in the group of normal elderly subjects respectively. $p=1$ represents the total tapping distance, $p=2, \ldots, 9$ signify the average and $\mathrm{CV}$ of maximum amplitude, finger tapping interval, maximum opening velocity and maximum closing velocity, and $p=10$ denotes the average zero-crossing number of acceleration. Each index for the normal elderly subjects follows a normal distribution as the average becomes 0 and the standard deviation becomes 1 .

The extracted features are also discriminated for operation of machines. In this Chapter, a log-linearized Gaussian mixture network (LLGMN) [22] is used as the PNN. This LLGMN is based on the Gaussian mixture model (GMM) and the log-linear model of the probability density function, and the a posteriori probability is estimated based on GMM by learning. Through learning, the LLGMN distinguishes movement patterns with individual differences, thereby enabling precise pattern recognition for bioelectric signals such as EMG and EEG [18]-[22].

In the training mode, the system first instructs the user to conduct $K$ types of finger tapping movement with different features, such as the amplitude of tapping and the opening velocity. The feature vectors calculated from these movements are then input to the LLGMN as teacher vectors, and the LLGMN is trained to estimate the a posteriori probabilities of each movement. After the training, the system can calculate the similarity between patterns in the user's movements and trained movements as a posteriori probabilities by inputting the newly measured vectors to the LLGMN. In order to prevent discrimination errors, the entropy $E(t)$ (which shows the obscurity of the information) is here calculated from the LLGMN outputs. Since the output $O_{k}(t)$ of the LLGMN represents a posteriori probabilities for each movement $M\left(M=M_{1}, M_{2}, \ldots, M_{K}\right)$, entropy is defined as

$$
E(t)=-\sum_{k=1}^{K} O_{k}(t) \log O_{k}(t)
$$

If $E(t)$ is smaller than discrimination determination threshold value $E_{d}$, the movement with the highest a posteriori probability becomes the result of discrimination. Otherwise, if $E(t)$ exceeds $E_{d}$, discrimination is suspended as obscure movement. Thus, the finger taps 
conducted by the user can be classified based on their features of movement using the LLGMN.

\subsection{Command encoding [24]}

The finger tapping movement of the user $M\left(M=M_{1}, M_{2}, \ldots, M_{K}\right)$ identified through LLGMN discrimination is allocated as operation command $U\left(U=U_{1}, U_{2}, \ldots, U_{c}\right)$, for each machine. $K$ denotes the number of movements conducted by the user, and $C$ represents the number of commands required to operate machines such as gaming consoles. Here, when the number of $K$ exceeds the total number of $C$, the corresponding estimated movement $M_{K}$ with command $U_{c}$ enables the user to directly execute commands using individual movements. However, since there are limits on the features of finger tapping movements that the user can voluntarily conduct, it is impossible to select all machine operation commands using movement $M_{K}$.

For control of domestic appliances, therefore, operation commands are arranged in a hierarchical structure to enable a range of operations by repeating the commands of execution and selection [18]. With this method, if two patterns (such as menu changes and menu selections) can be distinguished, the system can be operated appropriately.

An example of the interface screen based on GUI function for domestic appliances is shown in Fig. 4, and indicates that the screens of the three hierarchies are layered. Each hierarchy is displayed as one screen. The screen, which is suitable for use in living environments, is designed for intuitive operation. There are several selectable areas on the screen. The user can move from an upper hierarchy to a lower hierarchy by choosing the desired area, and the intended operation is then performed. As an example, Fig. 4 shows the process to turn on a television set using the two interface operations of execution and selection. First, the user repeats the execution command in the first layer, and the selection command is carried out in the area that contains the television. This action expands the first-layer selection area into the second layer, and the television, MD player and electric fan are displayed on the same screen. Once again, the user repeats the execution command and selects the television using the selection command. In the third layer, the television interface displays commands for options such as power supply, volume control and channel selection. Finally, the user selects the power supply command using the execution and selection commands.

On the other hand, in the case of game operation, commands are grouped and selected using movements. When the number $K$ of the user movements and the required number $C$ of commands are given, all commands are divided into $G$ groups with $(K-1)$ commands $(K \geq 2)$. The number $G$ becomes
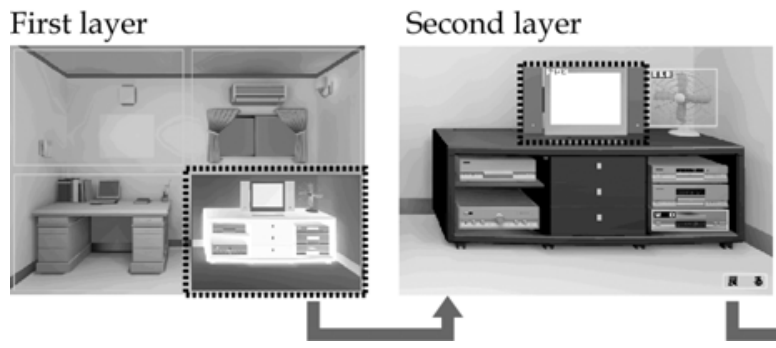

Third layer

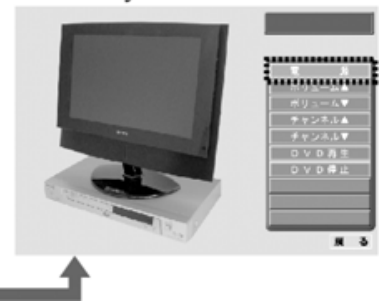

Fig. 4. GUI for domestic appliances 


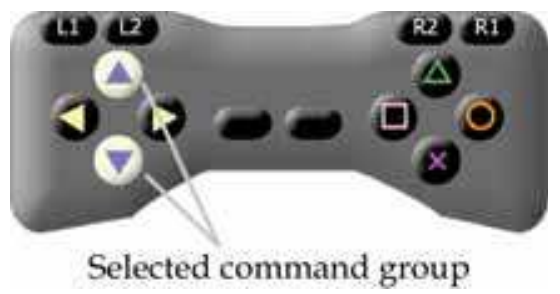

Fig. 5. GUI for game machines

$$
G=\operatorname{ceil}[C /(K-1)]
$$

where ceil $[y]$ is a function giving the minimum integer equal to or larger than real number $y$. The commands included in the group are freely configurable by the user, and can be set up in-line, e.g. increasing the number of commands based on the game machine in order to configure the same commands for multiple groups. The group can be changed using the remaining one of $K$ movements allotted to each group. Based on the above techniques, the user changes groups and selects commands by repeating $K$ movements.

Figure 5 shows an example of a GUI for game operation. This GUI uses a format similar to that of a game control pad, and the selected command group is displayed inverted. The user can therefore operate the game machine by shifting groups and selecting commands while watching the graphics on a monitor.

\subsection{Machine control [24]}

In general, since domestic appliances can be operated using IR communication, an IR transmitter and receiving unit [18] is utilized for the operation of each machine in this system. For domestic appliance operation, the IR signals corresponding to each command are set to the system in advance, and the user then controls each machine through selection of commands using finger tapping movements. In order for the IR unit to support the IR learning function [18], the IR signals of each appliance can be registered and deleted.

On the other hand, since gaming communication protocols differ from machine to machine, the system must be changed as needed. The game machine control circuit is therefore configured as a field-programmable gate array (FPGA) for easy reconstruction [19]. The FPGA, which is a large-scale integrated circuit (LSI), electrically reconfigures the internal circuit by rewriting the program. Less time is taken to implement the targeted circuit than through an application-specific integrated circuit (ASIC), allowing the program to be redesigned. In this system, a generation circuit to issue control signals corresponding to the selected command and a communication circuit to communicate with the game machine are implemented on an FPGA. The generation circuit uses a look-up table (LUT) to pre-store the control signals in the memory, to match the selected commands to the address in the memory, and to generate the required signal. The communication circuit includes the protocol of the individual game machine, and the control signals generated are sent to the machine according to IR signals received from the IR receiver attached to the FPGA. 


\subsection{Graphical output during evaluation [23]}

The measured signals, computed feature quantity and indices are displayed for doctors on a graphic display during evaluation of finger tapping movements. An example of the operation of the evaluation system is shown in Fig. 6. During operation, the monitor displays the following information: (i) the measured fingertip distance $d(t)$, velocity $v(t)$ and acceleration $a(t)$; (ii) computed indices and radar charts calculated for all measurement time and at prespecified time intervals; (iii) phase-plane trajectories of $d(t)$ and $v(t)$, and $v(t)$ and $a(t)$ on a real-time basis (the phase-plane trajectories can visually describe the dynamics of motion); (iv) operation buttons; and (v) a scrollbar to allow the waveform display time and the scale of the figure to be changed. Users can also input information and observations and use them for electronic medical charts and databases, which enables comparison with previous measurement data.

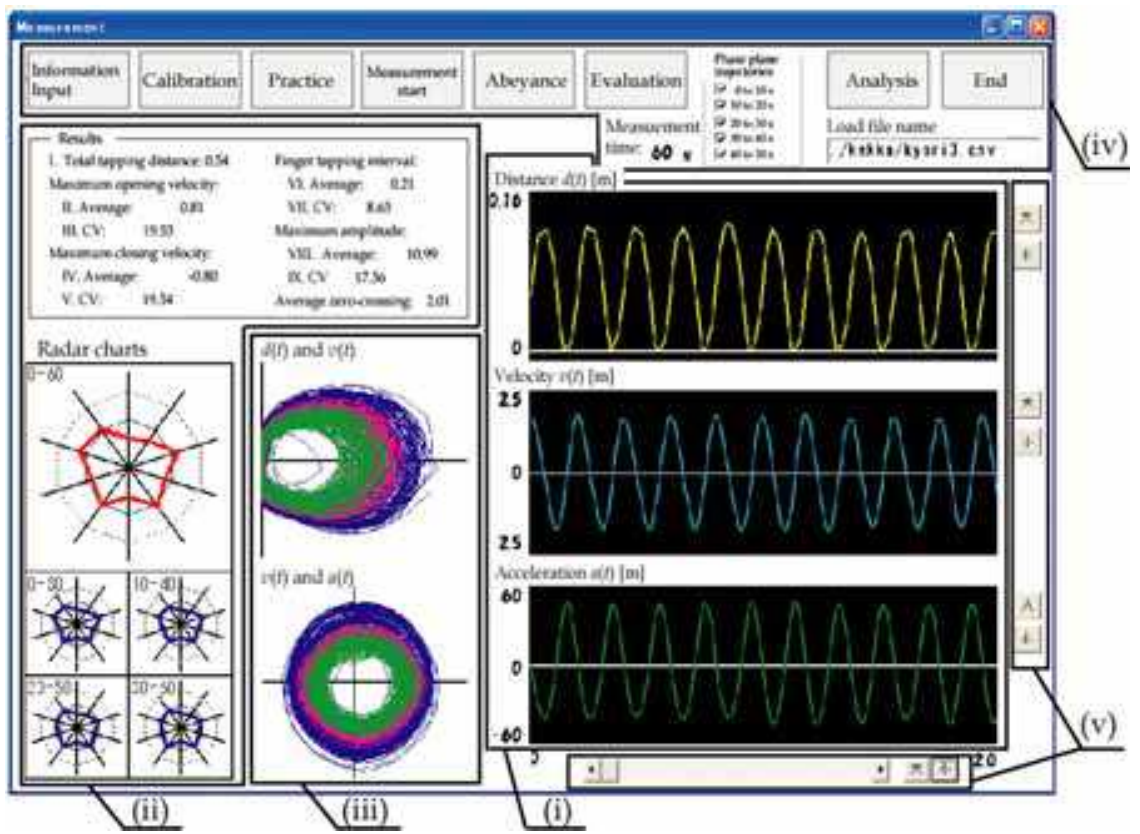

Fig. 6. Example of graphic display during evaluation [23] 


\section{Evaluation experiments of finger tapping movements}

To verify the validity of the proposed system, it is necessary to investigate the effectiveness of the following two criteria: (i) finger tapping evaluation to assess motor function, and (ii) finger tapping training. We therefore developed the prototype, and the conducted experiments involving evaluation and discrimination of finger tapping movements, operation of domestic appliances and a game machine, and finger tapping training using the developed prototype. The effectiveness of finger tapping evaluation is explained in this section, and the validity of finger tapping training is discussed in Section 4.

In the prototype system, the control circuit of the game machine is designed using an evaluation board (RC100, Celoxica) on which the FPGA (XC2S200-5FG456) is mounted, and the circuit is described using Verilog-HDL. The operation frequency of the circuit is 2.5 $[\mathrm{MHz}]$, and the control signal bit width stored in the memory is 16 bits. The LUT and communication circuits are implemented based on the communication protocol of the PlayStation 2 (Sony Computer Entertainment Inc., PS2), which the user operates using finger tapping movements.

\subsection{Experimental conditions}

The subjects were 16 patients with PD (average age: 71.2 6.4, male: 5, female: 11) and 32 normal elderly subjects (average age: 68.2 5.0, male: 16, female: 16). The subjects were directed to assume a sitting posture at rest. The coils were attached to the distal parts of the thumb and index finger as shown in Fig. 1, and the magnetic sensor was calibrated using three calibration values of 20,30 and $90 \mathrm{~mm}$. After a brief finger tapping movement trial using both the left and right hands, the movement of each hand was measured for $60 \mathrm{~s}$ in compliance with instructions to move the fingers as far apart and as quickly as possible. The subjects were isolated from the electrical supply of the PC. The severities of PD in the patients were evaluated by a neuro-physician based on the finger tap test of UPDRS [3]. The investigation was approved by the local Ethics Committee, and written informed consent was obtained from all subjects. The calculated indices were standardized on the basis of values obtained from the normal elderly subjects. The parameters of analysis were $\eta=0.1$ and $\zeta=5 \mathrm{~mm}$, and the sampling frequency was $100 \mathrm{~Hz}$.

\subsection{Results and discussion}

Examples of the finger tapping movements of a normal elderly subject (a) and a PD patient (UPDRS-FT 2: UPDRS part III Finger Tapping score 2) (b) are shown in Figs. 7. Figure 7 plots the measured fingertip distance $d(t)$, velocity $v(t)$ and acceleration $a(t)$. This figure shows the results of the measured data during the period from 0 to $10 \mathrm{~s}$. Further, a radar chart representation of the results of the indices is shown in Fig. 8; (a) to (c) illustrate the charts of normal elderly subjects, PD patients with UPDRS-FT 1 and those with UPDRS-FT 2 respectively. The solid lines describe the average number of normal elderly subjects, and the dotted lines show double and quintuple the standard deviation (2SD, 5SD) in Fig. 8. Further, in order to verify whether each index can evaluate Parkinsonian symptoms, the indices of PD patients and normal elderly subjects were compared using a heteroscedastic t-test. Table 1 shows the test results of each evaluation index. 


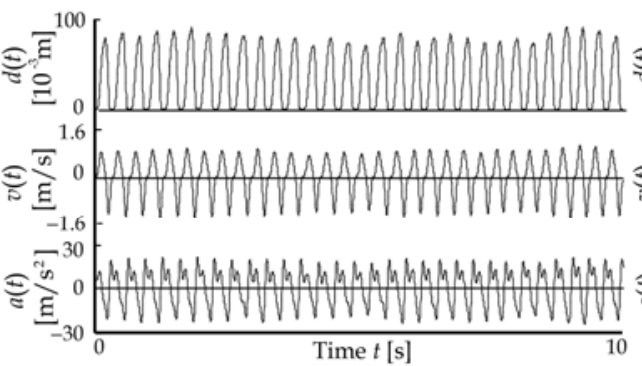

(a) A normal elderly subject

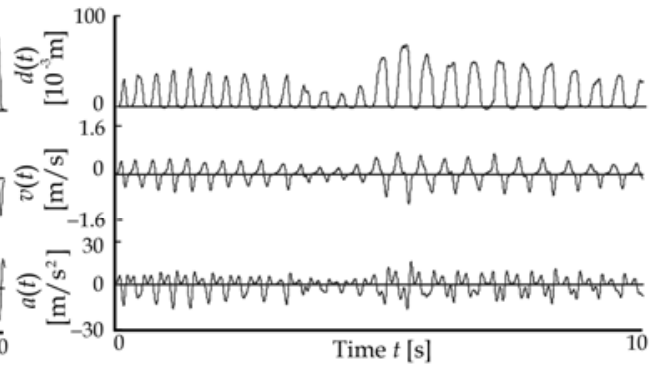

(b) A patient with Parkinson's disease (UPDRS-FT 2)

Fig. 7. Measured results of finger tapping movements [23]

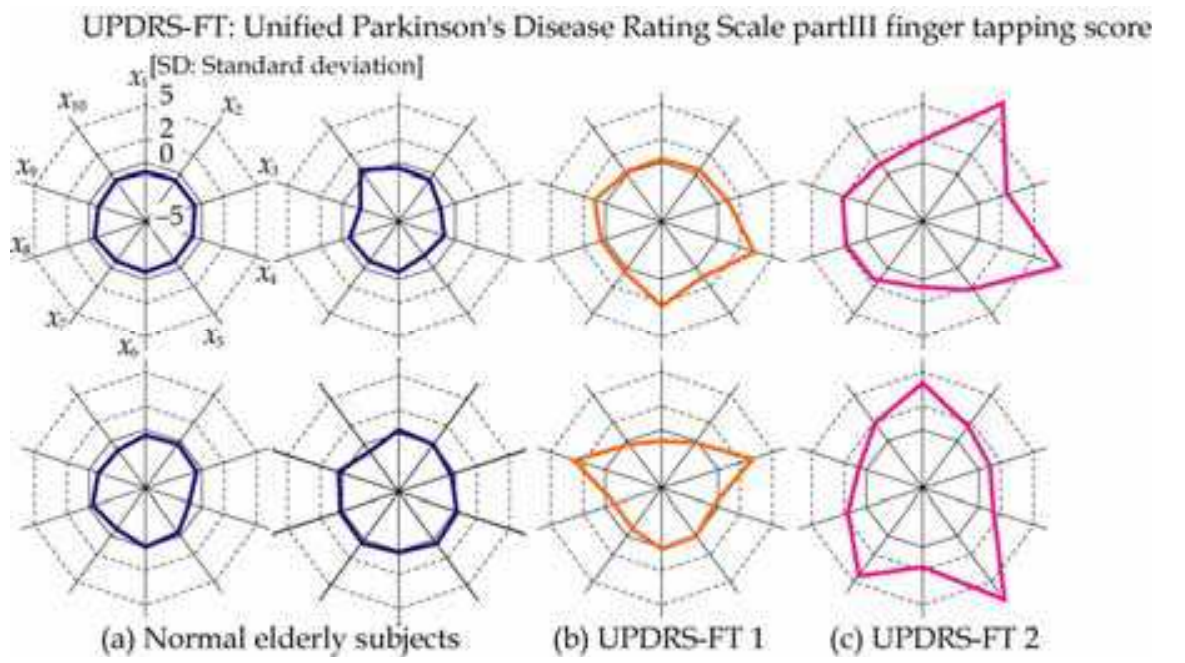

Fig. 8. Examples of radar chart representation of the results of the evaluated indices [23]

The experiments demonstrated that the movement waveforms of PD patients and normal elderly subjects have different tapping rhythms and scales, in which PD patients show larger variation in tapping rhythm and smaller scale than normal elderly subjects (Fig. 7). Further, by plotting radar charts of the indices of movements computed and standardized on the basic values obtained from normal elderly subjects, we identified that data from normal elderly subjects lie near the average, while those in PD patients' charts become larger according to the severity of their conditions. These results lead us to the conclusion that radar charts can comprehensibly present evaluation results and features of movement. Moreover, comparison of each index of PD patients and normal elderly subjects using a ttest shows that all indices differ significantly at the $1 \%$ level $\left(x_{1}\right.$ to $x_{3}$, and $x_{6}$ to $\left.x_{9}\right)$ or the $5 \%$ level $\left(x_{4}, x_{5}, x_{10}\right)$, and these results denote the same tendency mentioned in [9] and [10]. In the case of evaluating the severity of $\mathrm{PD}$, however, the indices differing significantly at the $1 \%$ level between UPDRS-FT 1 and -FT 2,-FT 1 and -FT 3, and -FT 2 and -FT 3 are only three 


\begin{tabular}{c||c||c||c||c}
\hline \multirow{2}{*}{$\begin{array}{l}\text { Evaluation } \\
\text { indices }\end{array}$} & \multicolumn{4}{c|}{ Significance probability $p$} \\
\cline { 2 - 5 } & Normal elderly and PD & UPDRS-FT1 and -FT2 & UPDRS-FT1 and -FT3 & UPDRS-FT2 and -FT3 \\
\hline \hline$x_{1}$ & $2.552 \times 10^{-4 * *}$ & $3.084 \times 10^{-2 *}$ & $1.024 \times 10^{-2 *}$ & $1.128 \times 10^{-1}$ \\
\hline$x_{2}$ & $9.345 \times 10^{-7 * *}$ & $2.751 \times 10^{-1}$ & $8.976 \times 10^{-2}$ & $1.062 \times 10^{-1}$ \\
\hline$x_{3}$ & $1.701 \times 10^{-5 * *}$ & $4.292 \times 10^{-5 * *}$ & $2.569 \times 10^{-3 * *}$ & $2.463 \times 10^{-1}$ \\
\hline$x_{4}$ & $3.694 \times 10^{-3 *}$ & $4.916 \times 10^{-1 *}$ & $2.869 \times 10^{-1}$ & $5.172 \times 10^{-1}$ \\
\hline$x_{5}$ & $1.265 \times 10^{-2 *}$ & $2.126 \times 10^{-1 * *}$ & $4.332 \times 10^{-2 *}$ & $8.354 \times 10^{-2}$ \\
\hline$x_{6}$ & $1.888 \times 10^{-10 * *}$ & $1.313 \times 10^{-1}$ & $3.218 \times 10^{-2 *}$ & $5.820 \times 10^{-2}$ \\
\hline$x_{7}$ & $7.564 \times 10^{-8 * *}$ & $1.107 \times 10^{-1}$ & $2.289 \times 10^{-2 *}$ & $8.890 \times 10^{-2}$ \\
\hline$x_{8}$ & $1.302 \times 10^{-11 * *}$ & $1.018 \times 10^{-1}$ & $4.477 \times 10^{-2 *}$ & $7.164 \times 10^{-2}$ \\
\hline$x_{9}$ & $1.943 \times 10^{-6 * *}$ & $1.988 \times 10^{-1 * *}$ & $7.016 \times 10^{-5 * *}$ & $4.491 \times 10^{-2 *}$ \\
\hline$x_{10}$ & $1.268 \times 10^{-2 *}$ & $1.344 \times 10^{-1}$ & $6.900 \times 10^{-2}$ & $4.695 \times 10^{-1}$ \\
\hline
\end{tabular}

$x_{1}$ : Total tapping distance

$x_{6}$ : Average maximum opening velocity

$x_{2}$ : Average maximum amplitude $x_{7}$ : CV of the maximum opening velocity

$x_{3}$ : CV of the maximum amplitude $x_{8}$ : Average maximum closing velocity

$x_{4}$ : Average finger tapping interval $x_{9}$ : $\mathrm{CV}$ of the maximum closing velocity

$x_{5}$ : CV of finger tapping interval $x_{10}$ : Average zero-crossing number of acceleration

CV: Coefficient of variation, ** : Significance level 1.0\%, *: $5.0 \%$

Table 1. T-test results of the evaluation indices [23]

$\left(x_{3}, x_{5}, x_{9}\right)$, two $\left(x_{3}, x_{9}\right)$ and zero, respectively. Since the number of PD experimental subjects (16) was small, it is necessary to investigate and improve the indices for accurate evaluation of the severity of PD with an increased number of subjects.

\section{Finger tapping training experiments}

We conducted operation experiments of the domestic appliances and a game console to identify the basic effectiveness of the proposed method for finger tapping training. In the experiments, the subjects (three healthy males, A-C, 23-25 years old) were directed to assume a sitting posture at rest. The coils were attached to the distal parts of the first finger and the index finger as shown in Fig. 1. The magnetic sensor was calibrated using three output voltages and fingertip distances $(20,30,90 \mathrm{~mm})$ (Eq. 1). The parameter for determining the contact time of the fingertips was $\beta=0.1$, with a measurement sampling frequency of $100[\mathrm{~Hz}]$. The game used in the experiment was Othello (SUCCESS Corporation), and consent was obtained from all subjects.

\subsection{Operation experiments}

To examine the effects of discrimination of finger tapping movements, discrimination experiments were conducted using finger taps measured from all subjects. In the experiments, the subjects were asked to conduct two types of movement with low and high velocities $(K=2)$ during a fixed time. For LLGMN learning, 20 sets of feature vectors extracted from these movements were randomly selected, and a total of 40 sets of patterns were used as teacher vectors. The subjects were then asked to repeat two types of movement alternately, and the tapping was measured during a 20-second period. There were five trials, and the discrimination determination threshold was $E_{d}=0.1$. 


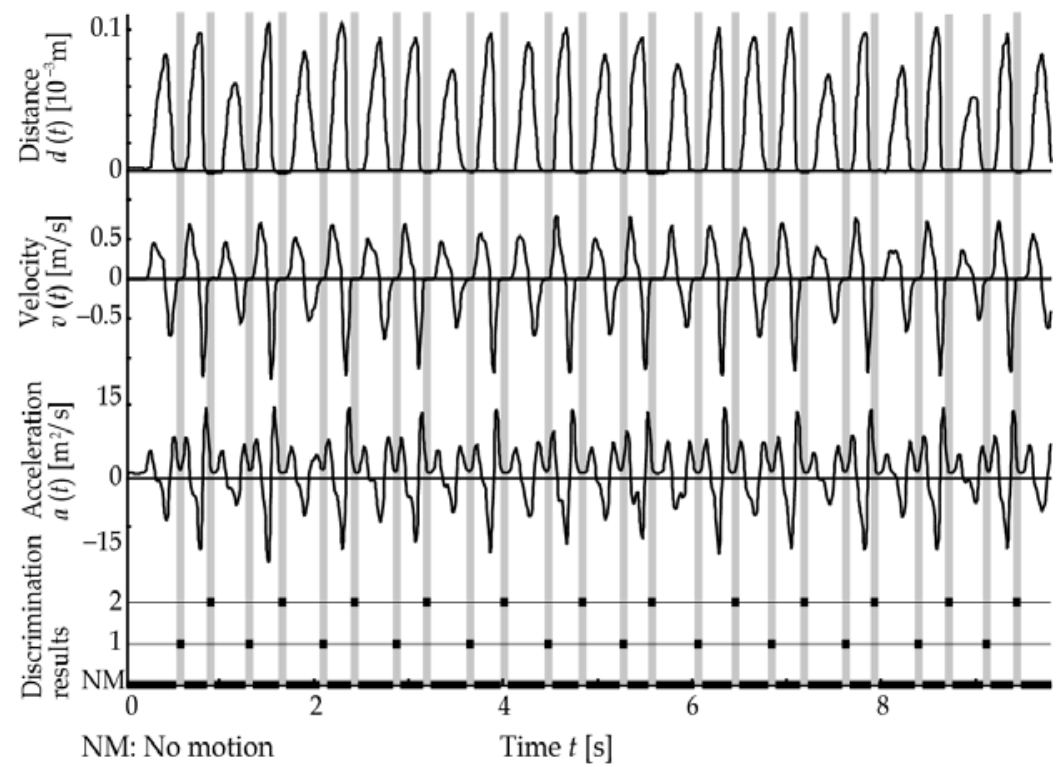

Fig. 9. An example of discrimination results [24]

An example of the results of finger tapping movement discrimination with subject $\mathrm{A}$ is shown in Fig. 9. This shows the plot of the measured fingertip distance $d(t)$ using a magnetic sensor, velocity $v(t)$, acceleration $a(t)$ waveforms and the discrimination results. The figure describes the results of the data measured during the interval 0-10 s. The shaded area indicates the contact time of the fingertips, and No motion (NM) in the discrimination results represents periods of no motion using Eq. 4. From the figure, it was confirmed that the subject performed low- and high-velocity movements iteratively, and that the movements were discriminated accurately by the system. The average discrimination rate of all trials with all subjects was $98.56 \pm 1.15$ [\%].

Experiments with domestic appliances and game operation were also conducted. In these experiments, the subjects were asked to operate the machines by voluntarily performing four types of finger tapping movement $(K=4)$ related to velocity and amplitude. These were $M_{1}$ (low velocity and small amplitude), $M_{2}$ (low velocity and large amplitude), $M_{3}$ (high velocity and small amplitude) and $M_{4}$ (high velocity and large amplitude). Instructions were given to operate each machine as presented in Fig.10. Figure 11 shows examples of the results of operation ((a) domestic appliance operation; (b) game operation), and includes fingertip distance $d(t)$, velocity $v(t)$, acceleration $a(t)$ waveforms, discrimination results, layers of menu and command groups, and selected commands. The shaded area indicates the contact time of the fingertips. It should be noted that two movements $\left(M_{1}\right.$ and $\left.M_{2}\right)$ were used for operation of domestic appliances, and four $\left(M_{1}\right.$ to $\left.M_{4}\right)$ were used for game operation. Here, the changing of groups by game operation commands was decided based on Fig. $12(C=14)$. Figure 11 shows that the subjects could operate each machine using finger tapping movements with different velocities and amplitudes. We therefore concluded 


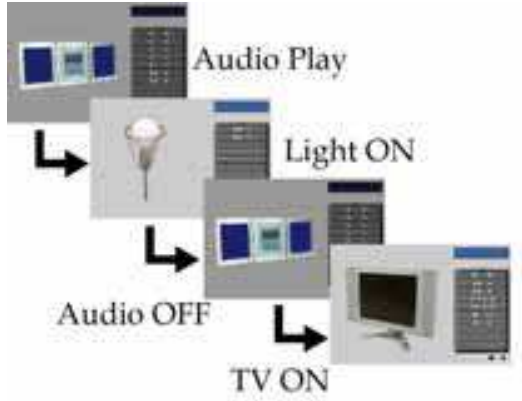

(a) Home electric appliances operation

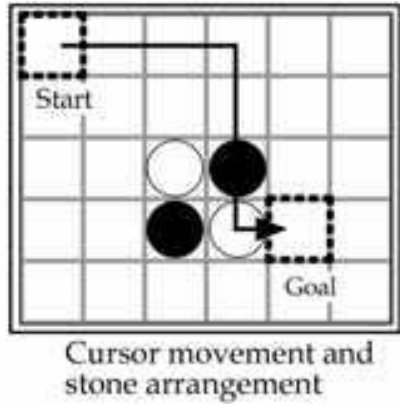

(b) Game operation

Fig. 10. The target tasks in the experiments [24]

that the subjects were able to voluntarily conduct finger taps and operate the machines as instructed.

\subsection{Example of the training experiments}

To identify the effectiveness of the developed interface for motor function training, training experiments were conducted on all subjects. After a brief trial, finger tapping movement was measured for $30 \mathrm{~s}$ with the instruction to move the fingers while maintaining values for the maximum amplitude of finger taps, the finger tapping interval, the maximum opening velocity and the maximum closing velocity. The average of each value and half the average value of the maximum amplitude of finger taps were then used as teacher vectors of class 1 and 2 respectively, and the LLGMN was trained $(K=2)$. The subjects could therefore operate the game using two types of movement ( $M_{1}$ : large amplitude of finger taps; $M_{2}$ : small amplitude). In other words, the subjects had to reproduce two types of trained movement for game operation. Further, they were instructed to play one game of Othello, and the movements were measured again after the game. The discrimination determination threshold was $E_{d}=0.1$.

Figure 13 shows the experimental results, and plots each subject's coefficient of variation $(\mathrm{CV})$ of each feature measured for $30 \mathrm{~s}$ before and after game operation. It is observed that the CVs of each value after the game are smaller than those before it. As the results indicate, the developed interface system is feasible for use in motor function training of finger tapping movements through game machine and domestic appliance operation. 


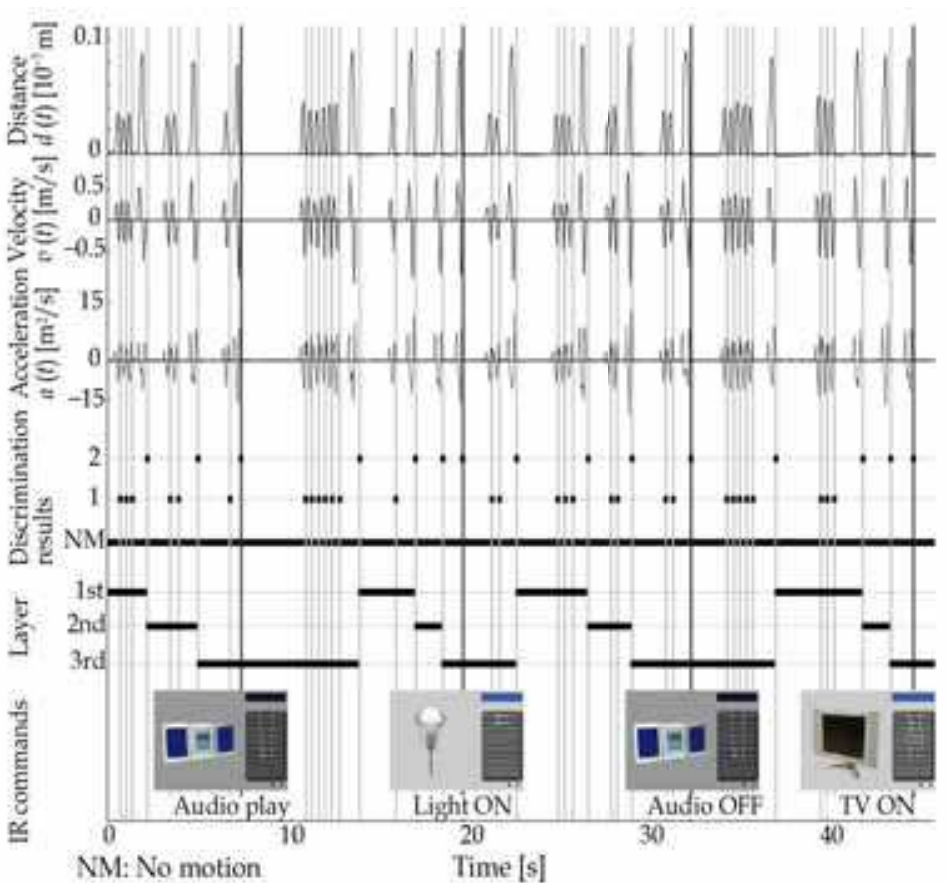

(a) An example of home electric appliances operation

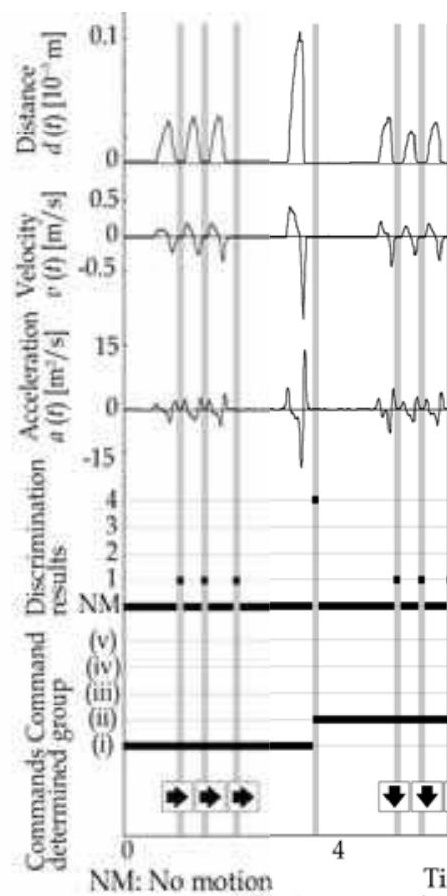

(b) An example

Fig. 11. An example of operations using the four types of finger tapping movements [24] 


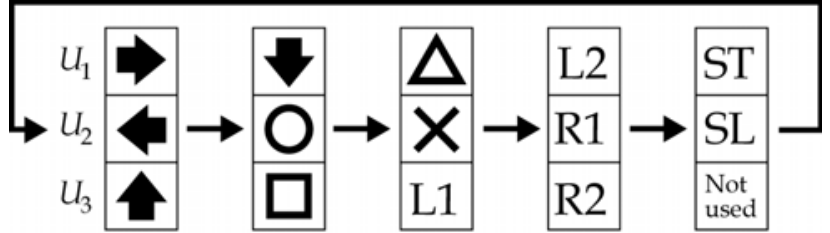

(i)

(ii)

(iii)

(iv)

(v)

Fig. 12. The command groups in the operation experiments [24]

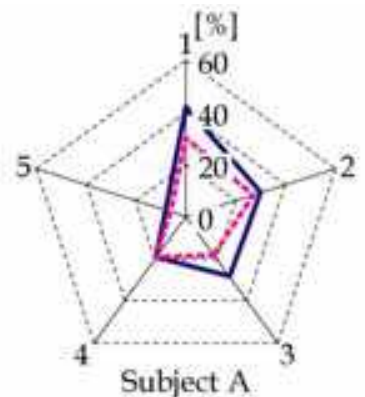

1: CV of maximum amplitude

2: CV of maximum opening velocity

3: $\mathrm{CV}$ of maximum closing velocity

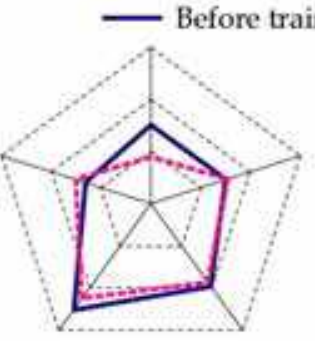

Subject B

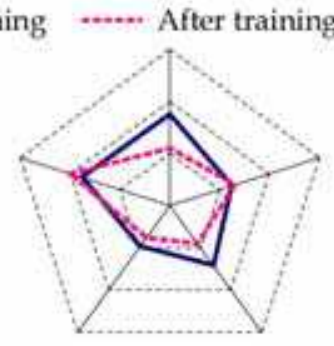

Subject C

$4: \mathrm{CV}$ of finger tapping interval

5:CV of zero-crossing number of acceleration

$\mathrm{CV}$ : Coefficient of variation

Fig. 13. Experimental results of training with each subject [24]

\section{Conclusion}

A movement evaluation and training system of finger tapping movements has been explained in this Chapter. The system involves the computation of ten evaluation indices measured from finger movements using magnetic sensors, and enables operation of game machines and domestic appliances for rehabilitation training.

The results obtained in the experiments using the prototype developed are summarized below.

- The average and coefficient of variance (CV) of the tapping interval $\left(x_{4}, x_{5}\right)$ and the average zero-crossing occurrences of acceleration $\left(x_{10}\right)$ in normal elderly subjects and those of Parkinson's disease patients differ significantly at the 5\% level, and the other indices differ significantly at the $1 \%$ level.

- The system was able to discriminate finger tapping movements voluntarily conducted by the subjects with high accuracy. The average discrimination rate was $98.56 \pm 1.15[\%]$ with all subjects.

- The subjects were able to operate the domestic appliances and game machine as instructed, and the subjects' finger tapping movements can be then evaluated in real time.

- In the case of finger movement training, the coefficient of variance in the features of each finger tap was reduced in comparison to before game-operation training. 
Our future research will involve improving the evaluation indices in order to enable diagnosis of the severity of the disease, as well as investigating the effects of aging with an increased number of subjects. We also plan to investigate the effects of training for patients with motor function impairment such as cerebrovascular disease using the proposed interface with an increased number of subjects, and to discuss adjusting the complexity of control tasks in domestic appliances and game machines for effective training.

Publications concerning this Chapter are listed in the bibliography [23],[24].

\section{Acknowledgements}

This study was supported in part by a Grant-in-Aid for JSPS Fellows (19.9510) from the Japan Society for the Promotion of Science.

\section{References}

Statistics and Information Department, Minister's Secretariat, Ministry of Health, Labour and Welfare: Patient survey, http://www.mhlw.go.jp/toukei/saikin/hw/kanja/ 05/index.html (in Japanese)

Parkinson's Disease Socity. The number of patients with Parkinson's disease, http://www.parkinsons.org.uk/about-parkinsons/whatisparkinsons/how-manypeople-have-parkinson.aspx

Fahn, S.; Elton, RL.; Members of The UPDRS Development Committee. (1987) Unified Parkinson's Disease Rating Scale, In: S. Fahn, CD. Marsden, DB. Calne, M. Goldstein, Recent Developments in Parkinson's Disease, Macmillan Health Care Information, vol. 2, pp. 153-304

Holmes, G. (1917). The symptoms of acute cerebellar injuries due to gunshot injuries, Brain, vol. 40, no. 4, pp. 461-535

Goetz, CG.; Stebbins, GT.; Chumura, TA.; Fahn, S.; Klawans, HL.; Marsden, CD. (1995). Teaching tape for the motor section of the unified Parkinson's disease rating scale, Movement Disorders, vol. 10, no. 3, pp. 263-266

Shimoyama, I.; Hinokuma, K.; Ninchoji, T.; Uemura, K. (1983). Microcomputer analysis of finger tapping as a measure of cerebellar dysfunction, Neurologia Medico Chirurgica, vol. 23, no. 6, pp. 437-440

Konczak, J.; Ackermann, H.; Hertrich, I.; Spieker, S.; Dichgans, J. (1997). Control of repetitive lip and finger movements in parkinson's disease, Movement Disorders, vol. 12, no. 5, pp. 665-676

Agostino, R.; Curra, A.; Giovannelli, M.; Modugno, N.; Manfredi, M.; Berardelli, A. (2003). Impairment of individual finger movements in Parkinson's disease, Movement Disorders, vol. 18, no. 5, pp. 560-565

Okuno, R.; Yokoe, M.; Akazawa, K.; Abe, K.; Sakoda, S. (2006) Finger taps acceleration measurement system for quantitative diagnosis of Parkinson's disease, Proceedings of the 2006 IEEE International Conference of the Engineering in Medicine and Biology Society, pp. 6623-6626 
Okuno, R.; Yokoe, M.; Fukawa, K.; Sakoda S.; Akazawa, K. (2007). Measurement system of finger-tapping contact force for quantitative diagnosis of Parkinson's disease, Proc. 2007 IEEE International Conference of the Engineering in Medicine and Biology Society, pp. 1354-1357

Kandori, A.; Yokoe, M.; Sakoda, S.; Abe, K.; Miyashita, T.; Oe, H.; Naritomi, H.; Ogata, K.; Tsukada, K. (2004). Quantitative magnetic detection of finger movements in parients with Parkinson's disease, Neuroscience Research, vol. 49, no. 2, pp. 253-260

Shima, K.; Kan, E.; Tsuji, Toshio; Tsuji, Tokuo; Kandori, A.; Miyashita, T.; Yokoe, M.; Sakoda, S. (2007). A new calibration method of magnetic sensors for measurement of human finger tapping movements, Transactions of the Society of Instrument and Control Engineers, vol. 43, no. 9, pp. 821-828 (in Japanese)

Thaut, M.H.; McIntosh, G.C.; Rice, R.R. (1997). Rhythmic facilitation of gait training in hemiparetic stroke rehabilitation, Journal of Neurological Sciences, vol. 151, pp. 207212

Enzensberger, W.; Oberlander, U.; Stecker K. (1997). Metronomtherapie bei ParkinsonPatienten, Der Nervenarzt, vol. 68, pp. 972-977

Del Olmo, M.F.; Arias, P.; Furio, M.C.; Pozo, M.A.; Cudeiro J. (2006). Evaluation of the effect of training using auditory stimulation on rhythmic movement in Parkinsonian patients - a combined motor and [18F]-FDG PET study, Parkinsonism and Related Disorders, vol. 12, pp. 155-164

Barea, R.; Boquete, L.; Mazo M.; Lopez, E. (2002). System for Assisted Mobility using Eye Movements based on Electrooculography," IEEE Trans. on Neural Systems and Rehabilitation Engineering, vol. 10, no. 4, pp. 209-218

Tanaka, K.; Matsunaga, K.; Wang, H. O. (2005). Electroencephalogram-Based Control of an Electric Wheelchair," IEEE Trans. on Robotics, vol. 21, no. 4, pp. 762-766

Shima, K.; Eguchi, R.; Shiba, K.; Tsuji, T. (2005). CHRIS: Cybernetic Human-Robot Interface Systems, Proceedings of 36th International Symposium on Robotics, WE1C3

Shima, K.; Okamoto, M.; Bu, N.; Tsuji, T. (2006). Novel Human Interface for Game Control Using voluntarily Generated Biological Signals, Journal of Robotics and Mechatronics, vol. 18 , no. 5, pp. 626-633

Fukuda, O.; Tsuji, T.; Kaneko M.; Otsuka, A. (2003). A Human-Assisting Manipulator Teleoperated by EMG Signals and Arm Motions, IEEE Trans. on Robotics and Automation, vol. 19, no. 2, pp. 210-222

Usui, S.; Amidror, I. (1982). Digital Low-Pass Differentiation for Biological Signal Processing, IEEE Trans. on Biomedical Engineering, vol. BME-29, no. 10, pp. 686-693

Tsuji, T.; Fukuda, O.; Ichinobe, H.; Kaneko, M. (1999). A Log-Linearized Gaussian Mixture Network and Its Application to EEG Pattern Classification, IEEE Trans. on Systems, Man, and Cybernetics-Part C: Applications and Reviews, vol. 29, no. 1, pp. 60-72

Shima, K.; Tsuji, T.; Kan, E.; Kandori, A.; Yokoe, M.; Sakoda S. (2008). Measurement and Evaluation of Finger Tapping Movements Using Magnetic Sensors, Proceedings of the 30th Annual International Conference of the IEEE Engineering in Medicine and Biology Society, pp. 5628-5631

Shima, K.; Tsuji, T.; Kandori, A.; Yokoe, M.; Sakoda S. (2008). A Tapping Interface for Finger Movement Training Using Magnetic Sensors, Proceedings of the 2008 IEEE International Conference on Systems, Man and Cybernetics (SMC 2008), pp. 2597-2602 


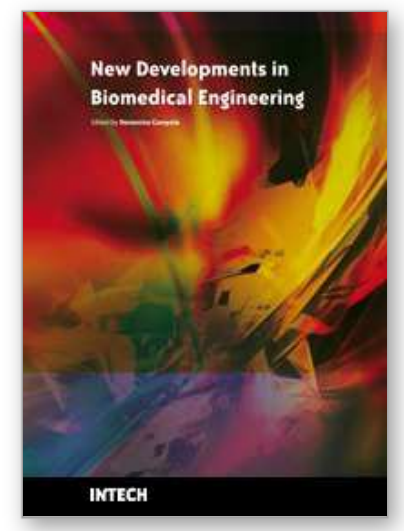

\author{
New Developments in Biomedical Engineering \\ Edited by Domenico Campolo
}

ISBN 978-953-7619-57-2

Hard cover, 714 pages

Publisher InTech

Published online 01, January, 2010

Published in print edition January, 2010

Biomedical Engineering is a highly interdisciplinary and well established discipline spanning across engineering, medicine and biology. A single definition of Biomedical Engineering is hardly unanimously accepted but it is often easier to identify what activities are included in it. This volume collects works on recent advances in Biomedical Engineering and provides a bird-view on a very broad field, ranging from purely theoretical frameworks to clinical applications and from diagnosis to treatment.

\title{
How to reference
}

In order to correctly reference this scholarly work, feel free to copy and paste the following:

Keisuke Shima, Toshio Tsuji, Akihiko Kandori, Masaru Yokoe and Saburo Sakoda (2010). Evaluation and Training of Human Finger Tapping Movements, New Developments in Biomedical Engineering, Domenico Campolo (Ed.), ISBN: 978-953-7619-57-2, InTech, Available from: http://www.intechopen.com/books/newdevelopments-in-biomedical-engineering/evaluation-and-training-of-human-finger-tapping-movements

\section{INTECH}

open science | open minds

\section{InTech Europe}

University Campus STeP Ri Slavka Krautzeka 83/A 51000 Rijeka, Croatia Phone: +385 (51) 770447

Fax: +385 (51) 686166 www.intechopen.com

\section{InTech China}

Unit 405, Office Block, Hotel Equatorial Shanghai No.65, Yan An Road (West), Shanghai, 200040, China 中国上海市延安西路65号上海国际贵都大饭店办公楼405单元 Phone: +86-21-62489820

Fax: +86-21-62489821 
(C) 2010 The Author(s). Licensee IntechOpen. This chapter is distributed under the terms of the Creative Commons Attribution-NonCommercial-ShareAlike-3.0 License, which permits use, distribution and reproduction for non-commercial purposes, provided the original is properly cited and derivative works building on this content are distributed under the same license. 